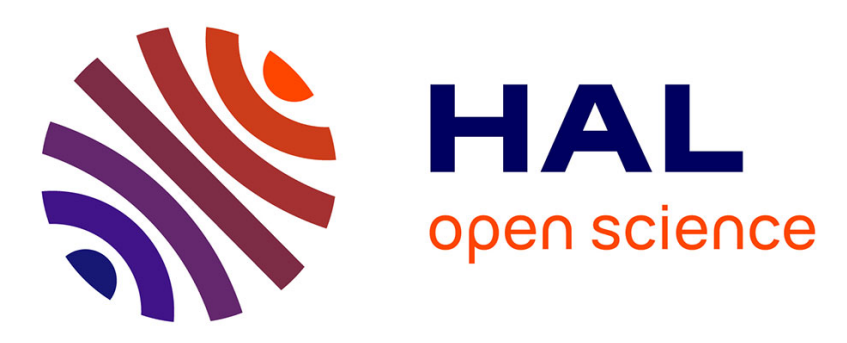

\title{
Particle optimization with metamodel for crack characterization
}

\author{
Rémi Douvenot, Marc Lambert, Dominique Lesselier
}

\section{To cite this version:}

Rémi Douvenot, Marc Lambert, Dominique Lesselier. Particle optimization with metamodel for crack characterization. URSI International Symposium on Electromagnetic Theory (EMT-S 2010), Aug 2010, Berlin, Germany. pp.887-890, 10.1109/URSI-EMTS.2010.5637338 . hal-00490009

\section{HAL Id: hal-00490009 \\ https://hal.science/hal-00490009}

Submitted on 7 Jun 2010

HAL is a multi-disciplinary open access archive for the deposit and dissemination of scientific research documents, whether they are published or not. The documents may come from teaching and research institutions in France or abroad, or from public or private research centers.
L'archive ouverte pluridisciplinaire HAL, est destinée au dépôt et à la diffusion de documents scientifiques de niveau recherche, publiés ou non, émanant des établissements d'enseignement et de recherche français ou étrangers, des laboratoires publics ou privés. 


\title{
Particle Optimization with Metamodel for Crack Characterization
}

\author{
Rémi Douvenot, Marc Lambert, Dominique Lesselier \\ Département de Recherche en Électromagnétisme \\ Laboratoire des Signaux et Systèmes, UMR 8506 (CNRS - Supélec - Université Paris Sud 11) \\ 3, rue Joliot-Curie 91192 Gif-sur-Yvette Cedex, FRANCE \\ firstname.lastnamedlss.supelec.fr
}

\begin{abstract}
A new inverse method for real time eddy current testing is introduced. It is based on particle swarm optimization coupled with a metamodel. This metamodel is generated by interpolating data from an adaptive database. Linear and radial basis function interpolations are compared as means to generate the metamodel. The inverse method gives a result combined with an approximation of the likelihood function to help for decision analysis. It is finally compared to a state-of-art inverse method using support vector machine on a 3D case on simulated data with very promising results.
\end{abstract}

\section{INTRODUCTION}

Eddy current testing (ECT) principle is to induce currents into a conductive piece, the pattern of which is modified by possible defects, inducing changes of impedance of a ECT probe nearby. So, defects can be detected and characterized from measurements of this impedance. In a number of cases, its application requires a database including many simulated cases for comparisons. Constructing this database might be too expensive if the number of parameters to describe the anomaly is high and if the database is contructed and used without proper strategy.

The few past years, great efforts have been made to generate adaptive databases for ECT [1], [2]. These databases minimize the number of points needed to fit the response surface by adding new points iteratively where the output is not properly approximated by the database. In consequence, in the final database, the faster the output varies, the denser the distribution of points is.

How to use the database is also crucial. The end-user needs in ECT are generally a fast response obtained in few seconds, and reliable results. Thus, the adaptive databases are usually employed to feed learning algorithms [3], [4] that give real-time inverse results. Also, one might bypass adaptive databases, via direct application of neural networks (NN) [5] or support vector machines (SVM) [6], [7]. This is investigated in [8] and the authors conclude that SVM are more efficient than NN for ECT.

A trade-off of learning algorithms is that they yield the inverted result without confidence interval or error probability, even if confidence learning machines could fill in this gap [9]. Therefore, another use is proposed for the adaptive database: a metamodel is created from the database, then a metaheuristic is applied to the inverse problem. The latter requires many forward simulations, which usually cannot be performed in reasonable time. Using the metamodel instead of the full simulations drastically decreases the computational load but degrades the accuracy of the inversion. This degradation depends on the accuracy of the metamodel. This is the reason for which it is at least as important as the metaheuristic itself.

\section{THE TEST CASE}

\section{A. Tube Properties}

The work piece under testing is a ferromagnetic tube characterized by a conductivity $\sigma_{\text {tube }}=3.5 \times 10^{6} \mathrm{~S} \mathrm{~m}^{-1}$ and imprecise relative permeability. There is air $\left(\sigma_{\text {air }}=\right.$ $\left.1 \times 10^{-10} \mathrm{~S} \mathrm{~m}^{-1}, \mu_{\mathrm{r}}=1\right)$ inside and outside of the tube. The latter is ideally infinitely extended along the $y$-axis with an internal radius equal to $r_{\text {tube }}=11.66 \mathrm{~mm}$ and a wall thickness $t_{\text {tube }}=4.29 \mathrm{~mm}$.

\section{B. Measurement System}

The measurement system is made of a transmitter bobbin of internal radius $r_{\mathrm{tx}}=7.75 \mathrm{~mm}$, thickness $t_{\mathrm{tx}}=2 \mathrm{~mm}$, height $h_{\mathrm{tx}}=15 \mathrm{~mm}$, and it is realized through 2700 wrappings. The probe is powered by a current $I_{0}=740.74 \mathrm{~A}$ at $f=150 \mathrm{~Hz}$. The receivers are a couple of bobbins of same shape as the one of the transmitter, but now characterized by an height $h_{\mathrm{rx}}=5 \mathrm{~mm}$ and involving 900 wrappings. They are set at $3 \mathrm{~mm}$ from each other and distant of $118 \mathrm{~mm}$ from the transmitter along the axis of the tube. The signal is obtained in a differential mode at $P=202$ different positions. The bobbins are inside the tube along the axial center and the set of complex measurements is collected from $y=0$ to $y=201 \mathrm{~mm}$ Therefore, the data to invert is a complex vector of dimension 202.

\section{Crack}

The crack is an external erosion of variable dimensions set at $y_{\text {pos }}=100.5 \mathrm{~mm}$. The defects are full grooves and therefore they have an angular extension fixed to $360^{\circ} \mathrm{C}$. They are characterized by $\sigma_{\text {crack }}=0 \mathrm{~S} \mathrm{~m}^{-1}, \mu_{\mathrm{r}}=1$. Their height and width are variables.

In that setting, the three parameters to invert are the internal radius (parameter P1) and width (parameter P2) of the crack, and the relative permeability of the tube (parameter P3). 


\section{Generation of THE DATABASE}

The procedure to generate the database is based on [10]. First an initial coarse mesh of simplices is defined. Then simulations are carried out at each node. The accuracy of each simplex is tested by comparing a simulation result with a linear interpolation at the middle of each edge of the simplex. If one interpolation is not accurate enough, a node is added at the middle of the longest edge of the simplex. The database used in this study is shown on the left of figure 1 during its generation at the $2^{\text {nd }}$ iteration ( 15 nodes) and in figure 2 at the $12^{\text {th }}$ and last iteration (101 nodes). The gray scale corresponds to the width, in order to emphasize depth in the 3D figure. At the right of the figures, the vectorial output data are plotted in a 2D space that preserves the distances using a multidimensional scaling [11]. This method allows to visualize high dimensional data in a low dimensional space. The axes have no physical meaning neither dimension, and they correspond to normalized distances

The aim of the adaptive database generation is to make it homogeneous in the output space. One can observe that during the generation, the homogeneity in the output space is made better. In consequence, there are areas in the input space where the points are denser, particularly here for high internal radii and widths and for low relative permeabilities, which corresponds to signals with the highest amplitudes.
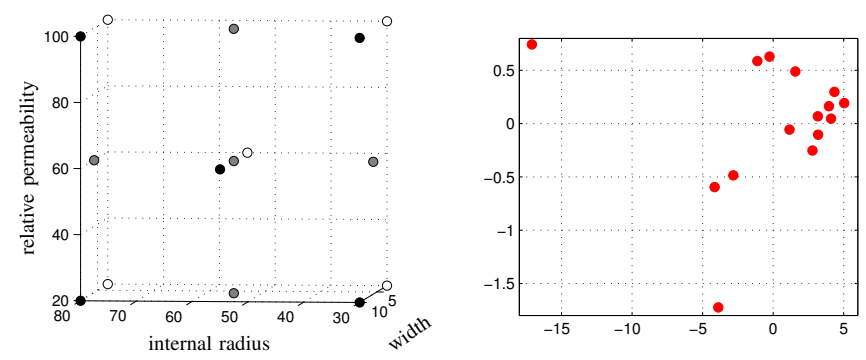

Fig. 1: Adaptive database at the $2^{\text {nd }}$ iteration (15 nodes) in the input (left) and output (right) space. The width is in $\mathrm{mm}$, the internal radius in $\%$ of the thickness of the tube, and the relative permeability is dimensionless.
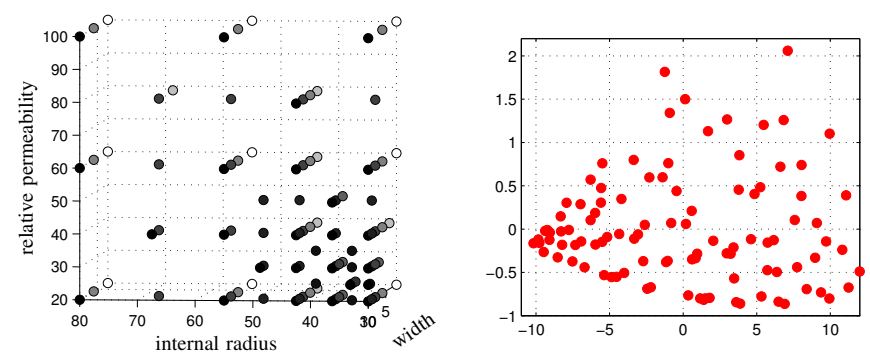

Fig. 2: Same as figure 1 at the $12^{\text {th }}$ iteration (101 nodes).

To visualize how the database tends to homogeneity with this method, it is compared to a database of same size (101 points) generated by Latin hypercube sampling (LHS) [12], plotted in figure 3 . The data generated by LHS are much less homogeneous in the output space and thus, it should be less efficient to approximate output from them. Some parts of the output space are oversampled and others are undersampled, something that adaptive databases tends to avoid.
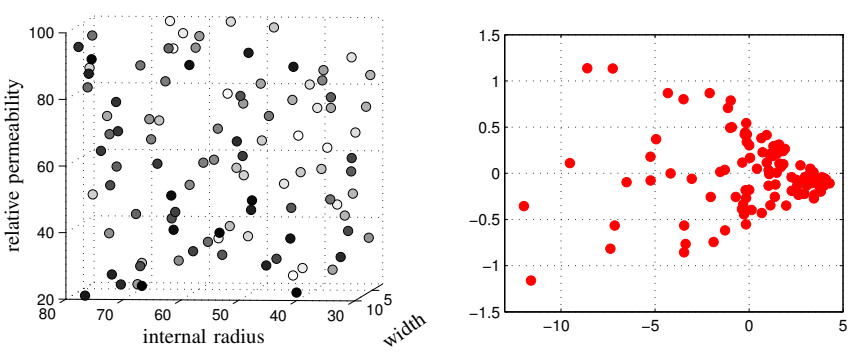

Fig. 3: Database generated by LHS (101 nodes) in the input (left) and output (right) space. The width is in $\mathrm{mm}$, the internal radius in \% of the thickness of the tube, and the relative permeability is dimensionless.

\section{MetAmodeL}

\section{A. Linear interpolation}

Two approaches to create the metamodel from the adaptive database are tested. The first, and more obvious, method is a linear interpolation. For each interpolated point, one looks for the simplex that contains the point and applies a linear interpolation from the vertices of the simplex. This interpolation method naturally follows the database generation.

\section{B. RBF interpolation}

An interpolation that is now using all points $\left(\mathbf{x}_{i}, \mathbf{y}_{i}\right)_{i=1, \ldots, N}$ of the database is the radial basis function (RBF) interpolation [13]. The principle is to express the vector to be interpolated, $\hat{\mathbf{y}}$, by

$$
\hat{\mathbf{y}}=\sum_{i=1}^{N} \mathbf{w}_{i} K\left(\mathbf{x}, \mathbf{x}_{i}\right)
$$

where $K$ is a kernel function [14], and the weights $\mathbf{w}_{i}$ are computed by learning on the training database. The thin plate spline (TPS) kernel is chosen. It is rather classical for data interpolation and since it has no intrinsic parameter, a tuning step is not required. The TPS kernel can be expressed as

$$
K\left(\mathbf{x}_{1}, \mathbf{x}_{2}\right)= \begin{cases}\left\|\mathbf{x}_{1}-\mathbf{x}_{2}\right\|^{2} \ln \left(\left\|\mathbf{x}_{1}-\mathbf{x}_{2}\right\|\right) & \text { if } \mathbf{x}_{1} \neq \mathbf{x}_{2} \\ 0 & \text { if } \mathbf{x}_{1}=\mathbf{x}_{2}\end{cases}
$$

\section{Comparing metamodels}

The normalized quadratic error of interpolation has been computed for 200 test cases picked up by LHS. The mean error is $1.27 \%$ for the linear interpolation and $0.24 \%$ for the RBF interpolation. More significant, the maximum error is $31.2 \%$ for linear interpolation and $2.1 \%$ for RBF interpolation. Actually, most points are well approximated by both methods, but linear interpolation may give poor results for some specific 
points, where the density is lower in the output space (figure 2).

One can also visually judge of the relevancy of the interpolation methods for the metamodel. In figure 4, 10 out of the 200 test cases are plotted in a low dimensional space using MDS (disks). In the same low dimensional space, the data obtained by linear interpolation (triangles) and RBF interpolation (crosses) are plotted. This figure illustrates the better accuracy of RBF and shows that the error is high with the linear interpolator where the distribution of points is of low density in the output space.

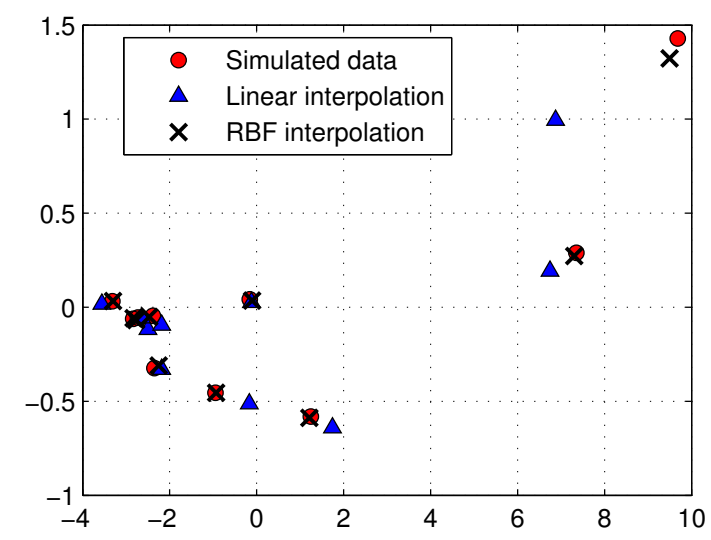

Fig. 4: In a low dimensional space, 10 simulated data with the approached data obtained by linear interpolation and by RBF interpolation.

\section{OPTIMIZATION METHOD}

The metamodels are used to feed an optimization algorithm. Particle swarm optimization (PSO) [15] under its Balanced PSO [16] variant is chosen for that purpose. The key idea of PSO is to put $M$ particles in the input space at positions $\mathbf{x}_{1}, \ldots, \mathbf{x}_{M}$. Then, they move on each dimension $d$ according to their best personal position $\mathbf{p}$ and their best local position 1 following the expression

$$
\begin{aligned}
& v_{d} \leftarrow w \times v_{d}+r_{1} c_{1}\left(p_{d}-x_{d}\right)+r_{2} c_{2}\left(l_{d}-x_{d}\right) \\
& x_{d} \leftarrow x_{d}+v_{d}
\end{aligned}
$$

The best local position is the best position that the particle shares with $k$ random particles. Here, as recommended in [15], $k$ is fixed to $3, w$ is set to 0.7 , and $c_{1}$ and $c_{2}$ are both set to 1.193. $r_{1}$ and $r_{2}$ are picked up uniformly into [0,1] at each iteration.

In its Balanced PSO variant, one particle moves to a local area of interest, which means around a local minimum. This version gives a satisfying compromise between exploration of the input space and exploitation of the area of interest [16].

\section{THE RESULTS OF INVERSIONS}

In the following, PSO-LIN denotes the PSO method with a metamodel that is obtained by linear interpolation and PSO$\mathrm{RBF}$ the same with RBF interpolation. The PSO results are compared to a SVM inversion. 200 configurations of defects are tested (the same cases as in section IV-C). We focus on the mean and maximum normalized quadratic error $\hat{q}$ and $q_{\max }$ on each parameter (P1, P2, and P3), and on the mean computation time $\hat{T}$.

TABLE I: Results obtained on the 200 test cases by PSO-LIN, PSO-RBF, and SVM.

\begin{tabular}{|l|ccc|ccc|ccc|}
\hline & \multicolumn{3}{|c|}{ PSO-LIN } & \multicolumn{3}{c|}{ PSO-RBF } & \multicolumn{3}{c|}{ SVM } \\
& P1 & P2 & P3 & P1 & P2 & P3 & P1 & P2 & P3 \\
\hline$\hat{q}$ & 0.8 & 2.8 & 0.5 & 0.6 & 1.9 & 0.5 & 0.5 & 0.6 & 0.2 \\
$q_{\max }$ & 22.1 & 74.4 & 4.7 & 9.3 & 22.7 & 23.1 & 4.2 & 14.8 & 1.7 \\
$\hat{T}(\mathrm{~s})$ & & 218.4 & & & 1.8 & & & $10^{-4}$ & \\
\hline
\end{tabular}

The use of RBF interpolation much improves the PSO method compared to the linear interpolation. Moreover, it is faster. Indeed, for linear interpolation the search of the simplex containing the input takes time. PSO-RBF, in a reasonable time, presents even comparable results with SVM. The advantage of PSO is that many points are explored during the optimization. Thus, an approximation of the likelihood function can be obtained. Meanwhile, SVM only gives the inversion results without any further information.

To illustrate how the PSO-RBF provides useful information, let us take two crack configurations as examples. The crack dimensions and results on these two cases are summarized in table II. Notice that crack 1 corresponds to $q_{\max }$ on P3 using PSO-RBF.

TABLE II: Crack configurations and results for the two examples.

\begin{tabular}{|l|ccc|ccc|}
\hline & \multicolumn{3}{|c|}{ Crack 1 } & \multicolumn{3}{c|}{ Crack 2 } \\
& P1 & P2 & P3 & P1 & P2 & P3 \\
\hline True parameters & 2.67 & 75.7 & 52.6 & 6.71 & 55.5 & 53 \\
Error $q(\%)$ & 0.02 & 0.01 & 23.1 & 0.03 & 0.24 & 0.00 \\
\hline
\end{tabular}

Figure 5 shows the likelihood functions obtained by PSORBF plotted with respect to the parameters in pairs. Only level lines are plotted. The circles indicate the true value of the parameters, and the squares indicate the maximum of the likelihood. On the left are plotted the results for the crack 1, and on the right for the crack 2 .

In the case of the crack 1, the true parameters P1 and P2 are very well approximated by PSO. For parameter P3, it is on the edge of the most likely area. The level lines spread on large areas, indicating that the uncertainty is high. Even if the inverted result is not accurate for parameter P3, the high uncertainty helps the end-user not to be too confident in the maximum of likelihood.

In the case of the crack 2, the inversion result is clear and one can be confident in it because the most likely areas are concentrated around the maximum of likelihood. Actually, the inverted results (crosses) are very close to the true parameters (squares). For this case, the method provides an accurate inversion with high confidence.

The presented inverse method is in general accurate and gives information on the reliability of the inversion. So, it is 

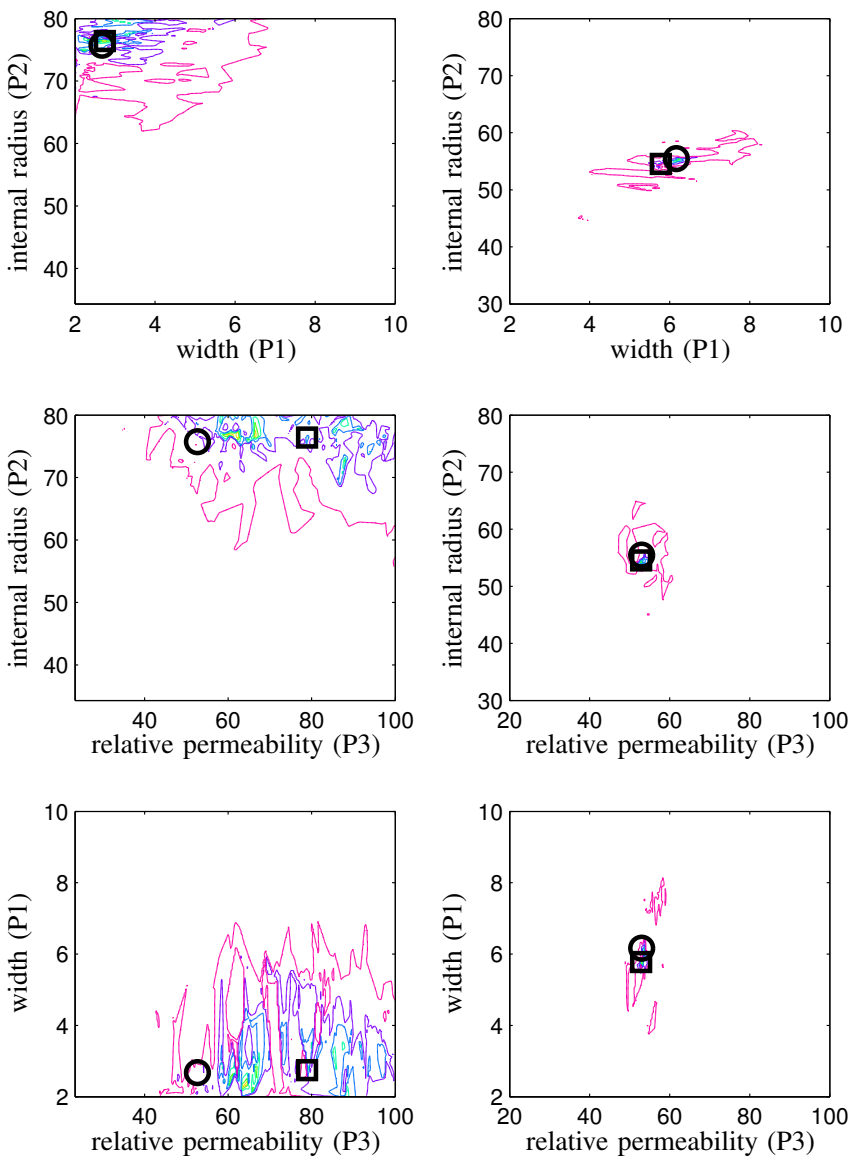

(a) Crack 1

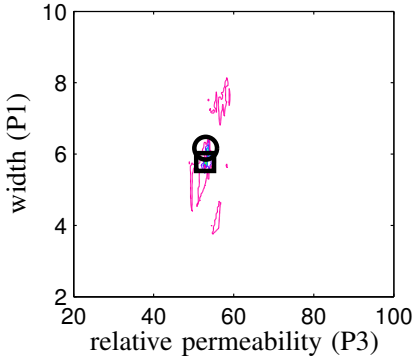

(b) Crack 2

Fig. 5: For the two studied cracks, level lines of the likelihood functions with respect to the input parameters in pairs. Real parameters are indicated with a circle, maximum of likelihood with a square.

useful as a decision analysis tool.

\section{CONCLUSION}

An original method to invert ECT signals has been presented. It is based on PSO combined with a metamodel. The method deals with ill-posedness. It highlights the indeterminate cases by providing an approximation of the likelihood function. It is illustrated on a specific 3D ECT problem with simulated data.

The use of adaptive generation helps to create efficient databases for metamodels since the output data are well approximated in the whole output space, unlike more classical methods as LHS. RBF is shown to be an efficient and

fast interpolation, even with vectorial data. So, employing a metamodel created from an adaptive database with RBF interpolation is an efficient method, particularly for vectorial data. It can be used to feed an optimization method, here PSO. During the optimization, the cost function is evaluated for many configurations, which enables to highlight ill-posedness, if any.

Work in progress includes the processing of laboratorycontrolled data.

\section{REFERENCES}

[1] J. Pávó and S. Gyimóthy, "Adaptive inversion database for electromagnetic nondestructive evaluation," NDT \& E International, vol. 40, pp. 192-202, Apr. 2007. [Online]. Available: www.sciencedirect.com/science/article/B6V4C-4MY0TRB1/2/a619013928abb1e5e569fa327bd13d31

[2] G. Franceschini, M. Lambert, and D. Lesselier, "Adaptive database for eddy-current testing in metal tubes," in Proc. of the 8th International Symposium on Electric and Magnetic Fields (EMF 2009), Mondovì, 26-29 May 2009, 2 pp. (CD-ROM).

[3] H. Acikgoz, L. Santandrea, Y. Le Bihan, S. Gyimóthy, J. Pávó, O. Meyer, and L. Pichon, "Generation and use of optimised databases in microwave characterisation," IET Sci. Meas. Technol., vol. 2, pp. 467-473, 2008.

[4] Y. Le Bihan, J. Pávó, and C. Marchand, "Characterization of small cracks in eddy current testing," Eur. Phys. J. Appl. Phys., vol. 43, pp. 231-237, 2008.

[5] D. J. MacKay, Information Theory, Inference, and Learning Algorithms. Cambridge University Press, 2003.

[6] V. Vapnik, The Nature of Statistical Learning Theory. New-York: Springer-Verlag, 1995.

[7] A. J. Smola and B. Schlkopf, "A tutorial on support vector regression," Royal Holloway Coll., Tech. Rep., 2003. [Online]. Available: http://eprints.pascal-network.org/archive/00002057/01/SmoSch03b.pdf

[8] A. Bernieri, L. Ferrigno, M. Laracca, and M. Molinara, "Crack shape reconstruction in eddy current testing using machine learning systems for regression," IEEE Trans. Instrum. Meas., vol. 57, pp. 1958-1968, 2008.

[9] H. Papadopoulos, K. Proedrou, V. Vovk, and A. Gammerman, "Inductive confidence machines for regression," in Proc. of the 13th European Conference on Machine Learning (ECML 2002), Helsinki, 19-23 Aug. 2002, pp. 345-356.

[10] M. Rivara, "Mesh refinement processes based on the generalized bisection of simplices," SIAM J. Numerical Analysis, vol. 21, pp. 604-613, 1984.

[11] S. M. Holland, "Non-metric multidimensional scaling (MDS)," Department of Geology, University of Georgia, Athens, Tech. Rep. GA 30602-2501, May 2008, 8 pp. [Online]. Available: www.uga.edu/strata/software/pdf/mdsTutorial.pdf

[12] W.-L. Loh, "On Latin hypercube sampling," Purdue Univ., Tech. Rep. 93-52, Oct. 1993. [Online]. Available: www.stat.purdue.edu/research/technical_reports/pdfs/1993/tr93-52.pdf

[13] M. D. Buhmann, Radial Basis Functions. Cambridge University Press, 2003.

[14] J. Mercer, "Functions of positive and negative type and their connection with the theory of integral equations," Philos. Trans. R. Soc. London, Ser. A, vol. 209, pp. 415-446, 1909.

[15] M. Clerc, Particle Swarm Optimization. London: ISTE, 2006.

[16] _ "The mythical balance or when particle swarm optimisation does not exploit," 2008, 6 pp. [Online]. Available: http://clerc.maurice.free.fr/pso/Balanced_PSO/Balanced_PSO.pdf 